\title{
"Mesopancreas-first" radical resection of pancreatic head cancer following the Cattell-Braasch-Valdoni maneuver: Appreciating the legacy of pioneers in visceral surgery
}

\author{
Konstantinos Diogeni Vasiliadis
}

Department of Surgery, Papageorgiou Hospital, Thessaloniki, Greece

\begin{abstract}
The "artery-first" approach pancreaticoduodenectomy, with maximal mesopancreas excision and central vascular ligation, represents the current principal determinants of radicality in pancreatic head cancer resection. However, these modifications at the resection stage of pancreaticoduodenectomy constitute extremely demanding and technically complicated procedures. Among the most critical contributing factors in the difficulty of artery-first approaches is the spiral configuration of the mesoduodenum and proximal mesojejunum around the superior mesenteric artery axis. This creates complicated tridimensional anatomy, making surgical dissection in the inferior peripancreatic anatomic area extremely challenging and demanding. The Cattell-Braasch-Valdoni maneuver (right-sided medial visceral mobilization and intestinal derotation maneuver) restores the embryological twist of the duodenojejunal junction, which demystifies the distorted peripancreatic vascular anatomy and facilitates a safe and radical "mesopancreas-first" pancreatic head cancer resection. The aim of this paper was to present the advantages, efficacy, and safety of the Cattell-Braasch-Valdoni maneuver in artery-first approach radical pancreaticoduodenectomy and provide a detailed description of its surgical technique.
\end{abstract}

Key Words: Pancreaticoduodenectomy; Mesenteric artery, superior; Pancreas; Pancreatic carcinoma; Dissection

\section{INTRODUCTION}

One of the principal concerns regarding the radicality of pancreatic head cancer resection is the strong tendency of pancreatic cancer cells to spread along the peripancreatic neural plexuses and infiltrate retropancreatic tissue [1]. This neurotropic retropancreatic spread of pancreatic head cancer is reflected in the high incidence of infiltrated retroperitoneal surgical margins (R1 resections) following a potentially curative Whipple's procedure, which has a significant negative impact on patient postoperative survival $[2,3]$. This has prompted calls

Received: May 7, 2021, Revised: June 1, 2021,

Accepted: June 2, 2021

Corresponding author: Konstantinos Diogeni Vasiliadis Department of Surgery, Papageorgiou Hospital, West Ring Road, Nea Efkarpia, GR-56 403, Thessaloniki, Greece

Tel: +30-2313-323556, Fax: +30-2310-670585, E-mail: konvasisurg@gmail.com ORCID: https://orcid.org/0000-0002-6964-060X

Copyright (C) The Korean Association of Hepato-Biliary-Pancreatic Surgery This is an Open Access article distributed under the terms of the Creative Commons Attribution Non-Commercial License (http://creativecommons.org/licenses/by-nc/4.0) which ermits unrestricted non-commercial use, distribution, and reproduction in any medium, provided the original work is properly cited. for a maximal or "complete" excision of the retropancreatic structures (the so-called mesopancreas) aiming at the radical margin-free excision of pancreatic head cancer, not only for localized cancers but also for cancers directly or indirectly invading the neighboring major vessels or regional lymph nodes [4-6].

Despite the documented [7] oncological importance of maximal mesopancreas excision, its precise anatomical definition remains an open issue given that it lacks well-defined anatomical boundaries, a fibrous sheath, and a fascial envelope. This explains the wide variety of often arbitrary and confusing terminologies for the mesopancreas in medical literature $[8,9]$. The International Study Group for Pancreatic Surgery (ISGPS) members [10] refrained from clearly defining the term and anatomical boundaries of the mesopancreas. Instead, they indecisively included its resection within the standard pancreatectomy definition, by using the term "lymphadenectomy". Regardless of the ISGPS members' point of view and despite conventional anatomical standards $[8,10]$, the mesopancreas is structured by the same histological components as that of a classical mesentery and is directly related to the ontogenetic evolution of the dorsal mesogastrium. Indeed, during embryo- 
genesis, the primitive peritoneal folds of the pancreas fuse with the retroperitoneal structures and finally disappear, unlike its contents (lymphatics, capillaries, blood vessels, and nerves), which remain in situ. These observations definitively present an embryologic rationale for the mesopancreas concept $[11,12]$. Moreover, a comprehensive perception of the surgical anatomy of the retropancreatic area is essential for ensuring anatomical precision in the delimitation of retropancreatic resection margins in pancreatic head cancer surgery. This constitutes a prerequisite for the standardization of the extent of mesopancreas excision as well as for the reproducibility of radical pancreatic head cancer surgery.

The lack of a commonly accepted, clear anatomical definition of the mesopancreas could be overcome by adopting the nomenclature provided by the Japan Pancreas Society as described in the 7th edition of the General Rules for the Study of Pancreatic Cancer [13]. Based on the aforementioned nomenclature, and according to the current practice of recognized surgical centers $[4,6,14]$, the retropancreatic structures that have to be excised during curative pancreaticoduodenectomy (PD) can be defined as the well-vascularized and nerve-rich lamina connecting the head of the pancreas to the right celiac ganglion and superior mesenteric artery (SMA) extending to the aortocaval groove. This anatomical area corresponds to the first and second nerve plexuses of the pancreatic head (plPh-I and plPh-II, respectively), which additionally encompasses the lymphatics (nodes and vessels), adipose tissue, capillaries, blood vessels (inferior pancreaticoduodenal artery [IPDA] and vein), and the uppermost mesojejunum with its corresponding jejunal arteries (JAs) and veins (JVs) [15]. Whether the loose connective tissue of the aortocaval groove should be regarded as a part of the mesopancreas remains a matter of controversy. Indeed, a number of pancreatic surgeons incorporate the para-aortic area (areas corresponding to $16 \mathrm{a} 2$ and 16b1 lymph node basins) in the resected retropancreatic tissue, arguing that a maximized excision of the retropancreatic neurovascular bundle between the SMA and celiac trunk along with the circumferential dissection of the SMA can achieve an adequate retropancreatic margin of clearance, which potentially controls cancer spread and results in a higher $\mathrm{R} 0$ resection rate [16].

During the last decades, the mesopancreas concept has led to the development of several technical modifications of PD at its resection stage, featuring maximal or "complete" mesopancreas excision. Concurrently, the artery-first approaches have been advocated, providing not only maximal mesopancreas excision but also early dissection of the SMA from the tumor, which ultimately confirms resectability before an irreversible step of the operation is undertaken (pancreas or bile duct transection) [1720]. The additional advantages of the SMA-first approaches are the secure identification and safeguarding of an aberrant right hepatic artery and early ligation of the IPDA. The latter prevents venous congestion of the pancreaticoduodenal complex before its resection, which significantly reduces intraoperative blood loss and fulfills the oncologic principle of the no-touch isolation technique $[19,21,22]$.

Regardless of the recognized advantages of the artery-first approaches and apart from the required compliance with the established principles of surgical oncology, it should be emphasized that the early identification and ligation of the inferior peripancreatic vessels with maximal mesopancreas excision are demanding and technically complicated procedures. This is not only because of the complicated and variant peripancreatic vascular anatomy but also because of the anatomical diversity of the pancreaticoduodenal complex, resulting from the developmental rotation processes of the midgut during embryogenesis. This results in a torsional arrangement of the pancreaticoduodenal complex and uppermost jejunum around the SMA axis. Respectively, the mesopancreas during embryogenesis rotates from the right-posterior to the left and forms a spiral around the SMA, becoming the central structure of mesenteric distortion [12]. This anatomic complexity is further compounded by the fact that during embryogenesis the mesopancreas fuses with the retroperitoneal structures, forming a complete and boundless anatomic continuum throughout the para-aortic area $[9,23]$. Hence, this creates a complicated tridimensional vascular anatomy around the ligament of Treitz, the proximal mesojejunum, and the pancreaticoduodenal complex. These features added to the fact that in most of the artery-first approaches, the mesopancreas is dissected with the upper jejunum left in situ, makes surgical dissection in the $\mathrm{plPh}-\mathrm{II}$ anatomic area extremely challenging and demanding. Incautious mobilization of the pancreatic uncus from the SMA or approach to the IPDA can be complicated with serious venous injury and bleeding, which can easily escalate to intractable hemorrhage, the management of which can jeopardize the integrity of the superior mesenteric vein (SMV) per se, or compromise the venous return of the proximal jejunum, which is planned for use in reconstruction. Accordingly, it becomes evident that the artery-first approaches, combined with maximal mesopancreas excision require deep insight into the surgical anatomy, dedication, and mastering of surgical techniques, and probably, accurate preoperative anatomical mapping regarding the anatomy of the inferior peripancreatic blood vessels. Synopsizing, the torsional arrangement between the pancreaticoduodenal complex and uppermost jejunum around the SMA, in addition to the complex tridimensional and variant anatomy of the inferior peripancreatic vessels, are among the most critical contributing factors in the difficulty of either conventional or artery-first approach PD (Fig. 1).

The implementation of the intestinal derotation maneuver in mesopancreas-first approach PD can serve as a means of demystifying the anatomical complexity of the inferior peripancreatic area. This master surgical technique, known as the Cattell-Braasch-Valdoni maneuver [24] derotates the primary umbilical loop in a 270-degree clockwise direction and restores the embryologically twisted duodenojejunal junction by trans- 


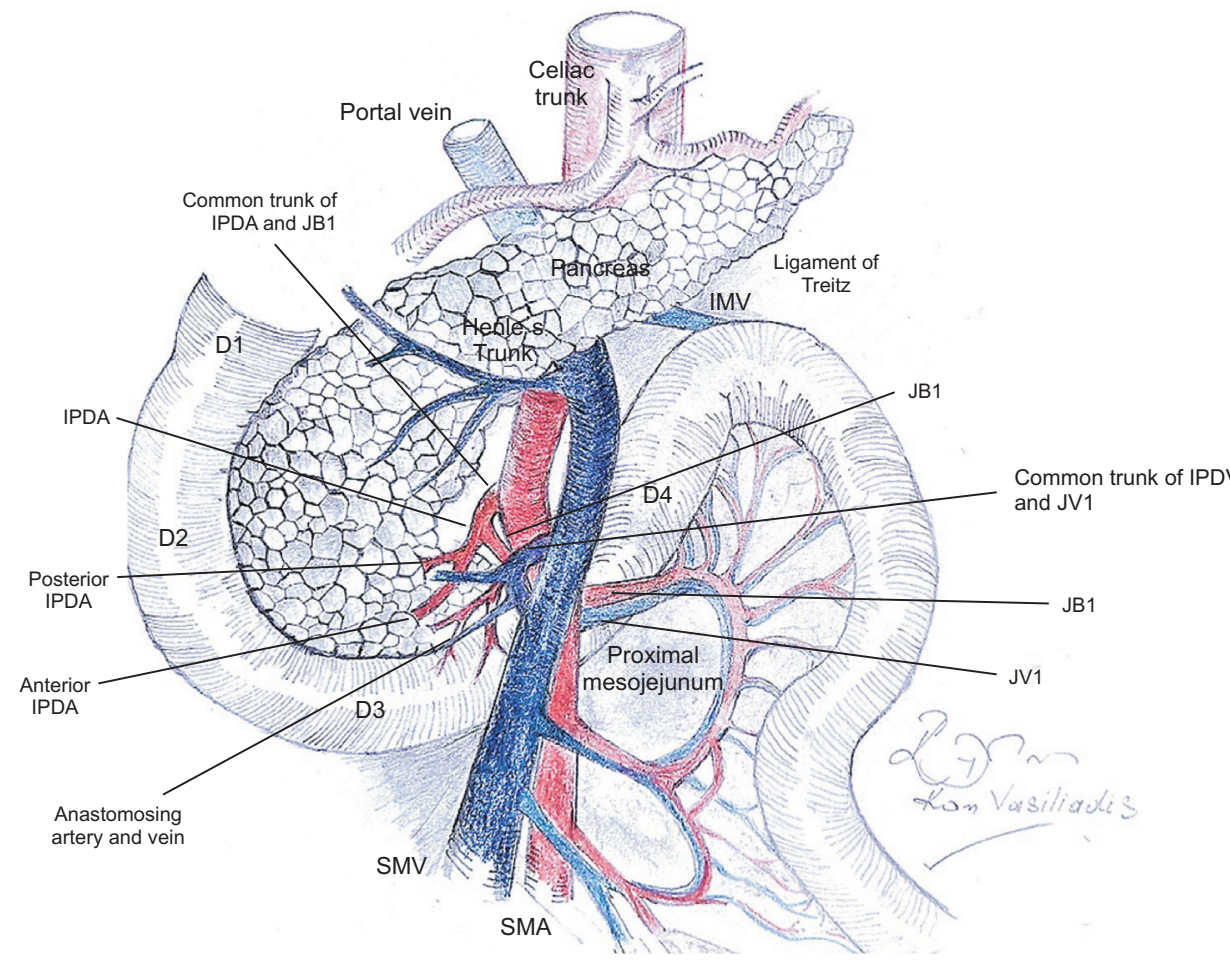

Fig. 1. Usual pattern of the vascular anatomy around the pancreatic head. The torsional arrangement between the pancreaticoduodenal complex and the uppermost jejunum around the superior mesenteric artery creates complicated tridimensional vascular anatomy. Additionally, there is a great chance of possible variations, making the inferior peripancreatic vascular anatomy consistently inconsistent. IMV, inferior mesenteric vein; IPDA, inferior pancreaticoduodenal artery; JB1, first jejunal branch; IPDV, inferior pancreaticoduodenal vein; JV1, first jejunal vein; SMV, superior mesenteric vein, SMA, superior mesenteric artery. posing the duodenum and uppermost jejunum to the right of the SMV-SMA axis in a two-dimensional horizontal plane. This maneuver simplifies and clarifies the distorted and complicated peripancreatic vascular anatomy, which facilitates safe and radical mesopancreas-first pancreatic head cancer resection. These advantages can provide a rational basis for applying the right-sided medial visceral mobilization and intestinal derotation maneuver in artery-first approach PD.

The aim of this study was to present the advantages, efficacy, and safety of the Cattell-Braasch-Valdoni maneuver in the radical resection of pancreatic head cancer and provide a detailed description of its surgical technique.

\section{VASCULAR ANATOMY OF THE MESOPANCREAS}

Artery-first approaches for pancreatic head cancer resection demand a thorough knowledge of peripancreatic vascular anatomy. However, standard textbooks and atlases of surgery fail to provide an accurate description of the surgical anatomy of the peripancreatic area. Furthermore, the available information on the surgical anatomy of proximal jejunal vessels and SMV tributaries is limited and confusing.

Accurate knowledge of peripancreatic surgical anatomy can be extracted from a number of very important, recently published clinical [6,25-27] and radiologic studies [28,29]. The latter, nicely describe the anatomy of peripancreatic and proximal jejunal vessels using three-dimensional computed tomography imaging. These radiologic studies apart from providing valuable information on the vascular anatomy of the mesopancreas, also underline the importance of individualized, precise, and detailed preoperative mapping of the peripancreatic and uppermost jejunal vascular anatomy in the surgical treatment of pancreatic head cancer.

\section{INFERIOR PANCREATICODUODENAL AND FIRST JEJUNAL ARTERY}

One of the crucial surgical steps of artery-first approach $\mathrm{PD}$ is early dissection and the control of blood flow into the pancreaticoduodenal complex and uppermost jejunum. This requires early dissection and division of the IPDA and the first jejunal artery (JA1) and depending upon cancer spread, the JA2 [6]. It is important to emphasize that the branching pattern of the IPDA and proximal JAs is considerably variant. Nevertheless, according to its usual branching pattern, the IPDA shares a common trunk (first jejunal arterial trunk, FJAT) with the JA1, which originates directly from the posterior aspect of the SMA [30,31].

However, in a significant proportion of cases, the IPDA arises directly from the SMA. In this variation, the IPDA originates from the posterior to the right aspect of the SMA, just cranial from the origin of the JA1. In this anatomical pattern, according to Inoue et al. [6], the IPDA usually originates on the dorsal aspect of the SMA between the 5 and 7 o'clock positions. In rare occasions, the anterior IPDA (aIPDA) and the posterior 
IPDA (pIPDA) originate directly from the posterior aspect of the SMA (absent an IPDA stem) or they can have different origins (aIPDA arising from the FJAT and PIPDA from the SMA) $[6,30,31]$. In extremely rare cases, the IPDA stem may originate from a replaced right hepatic artery arising from the SMA [32].

Also crucial, is a thorough understanding of the arterial anatomy of the uppermost mesojejunum. Usually, as mentioned above, the JA1 originates from the FJAT or directly from the posterior aspect of the SMA, just caudal from the origin of the IPDA. However, the chance of minor but dangerous arterial variations must be appreciated when dissecting the proximal mesojejunum. In fact, as shown in the study of Kobayashi et al. [33] on rare occasions, multiple JAs (JA1, JA2, and JA3) may originate from the FJAT (types Ib and Ic) or multiple JAs (JA1, JA2, JA3, and JA4) can share a common trunk directly originating from the SMA (types IIb, IIc, and IId).

Apart from the significant branching pattern variability of the IPDA and proximal JAs, it is very important to be aware that the SMA courses unexpectedly close to the level of its origin, lying left posteriorly and in immediate proximity to the posterior aspect of the SMV. Additionally, at this level, the mesentery of the uppermost jejunum (containing the root of the IPDA and JA branches) is short, vague, and distorted. These features make surgical dissection and clarification of the complex anatomy in the $\mathrm{plPh}-\mathrm{II}$ anatomic area a real challenge.

\section{INFERIOR PANCREATICODUODENAL VEIN}

According to classical anatomical standards, the common trunk emerging from the confluence of the anterior inferior pancreaticoduodenal vein (aIPDV) and the posterior IPDV (pIPDV) designates the IPDV. However, its definition is obscure in the medical literature. This is due not only to the branching pattern variability of the inferior pancreatic veins, but also the poor visualization of these veins on multidetector computed tomography imaging (because of their small diameter, complex anatomic relationship, and oblique course) and operative factors such as the anatomical complexity and limited surgical view that hinder a detailed intraoperative clarification of the vascular anatomy of the mesopancreas [34].

Usually, and contrary to many illustrations of the vascular anatomy of the pancreas in standard surgery textbooks, the pIPDV, running at the posterior aspect of the uncinate process and across the dorsal side of the SMV, drains into the first jejunal vein (JV1) or the first jejunopancreatic vein (FJPV), (which emerges from the joining of the pIPDV and various numbers of JVs and terminates in the posterior aspect of the SMV) [32]. The aIPDV, which runs between the third portion of the duodenum and the anterior aspect of the uncinate process, terminates directly on the posterior side of the SMV. In a minor but important variation, the FJPV is absent and the JV1 (or the common stem of a variant number of JVs), running across the ventral aspect of the SMA drains into the left aspect of the SMV. In this variation, the PIPDV and aIPDV drain independently, the former directly into the posterior surface of SMV and the latter into the anterior surface of the SMV [28]. Of note, during the surgical dissection of the mesopancreas, the IPDV is rarely identified. Indicative of the confusion regarding the definition of the IPDV is that most investigators define the IPDV as the main drainage vein of the uncinate process, regardless of intraoperative clarification of the anatomy and identification of the aIPDV and pIPDV [28] Additional, minor but not negligible variations in the course, branching pattern, and termination of the aIPDV and pIPDV further increase the surgical complexity of artery-first approach PD $[32,35]$.

\section{PROXIMAL JEJUNAL VEINS}

Comprehensive knowledge of the branching pattern of the distal SMV and proximal JVs is an additional prerequisite for safely performing an artery-first approach PD. However, again, as in the case of inferior pancreaticoduodenal veins, limited information is available on the network of the venous outflow of the uppermost jejunum, and especially the surgical anatomy of the JV1 and JV2. Furthermore, there is no standardized nomenclature for the proximal JVs, which are usually defined according to the branching order of the accompanying JAs. Notwithstanding, the vascular anatomy of the proximal mesojejunum is of great importance, given that radical PD necessitates the central ligation of JA1 and JV1 and mesenteric division along the line of JA2 and JV2.

According to their prevailing branching pattern, the JV1 and the JV2 form a common trunk that terminates into the posterior aspect of the SMV. The definition of the common trunk formed by the proximal JVs is also confusing and varies in the literature $[27,29,36]$

Ishikawa et al. [28] designated the common trunk of the JV1 and the JV2 as the first jejunal trunk (FJT). In this study, the authors reported that in $84 \%$ of patients, the FJT is formed by the joining of the JV1 and the JV2 and drains the territories of the JA1 and JA2, while in 39\% of patients, the FJT has a large diameter and drains more than two arterial territories (to the third, fourth, or fifth JAs). In contrast, Negoi et al. [32] designated the common trunk emerging from the confluence of the IPDVs and the JV1 or multiple proximal JVs as the jejunopancreatic trunk. Regardless of the confusing nomenclature of the common trunk formed by the proximal JVs, it is very important to be aware that this branch usually drains a broad territory of the proximal mesojejunum corresponding to several proximal JAs, which obviously has significant implications at the resection phase of the uppermost mesojejunum.

Equally important is the position of the FJT relative to the SMA. Particularly, the FJT usually runs dorsal to the SMA and terminates into the posterior aspect of the SMV but it can run ventrally to the SMA, terminating either into the SMV or the 
splenoportal confluence. It is very important to be aware of a ventrally located FJT as the technical difficulty of artery-first approaches is intensified. This is due to the poor surgical view of the SMA, which is hidden by the FJT, making the orifices of the IPDA and JA1 along the posterior aspect of the SMA practically inaccessible. Additionally, in a minor variation, the proximal JVs can terminate separately into the SMV, having a variable position relative to the SMA $[28,32]$ The complicated, understudied, and variant branching patterns of the venous network of the uppermost mesojejunum further escalates the surgical difficulty of artery-first approaches.

\section{SURGICAL MESOPANCREAS-FIRST PANCREATICODUODENECTOMY TECHNIQUE FOLLOWING THE \\ CATTELL-BRAASCH-VALDONI MANEUVER FOR THOSE WHO VALUE PRECISE, ONCOLOGICAL SURGICAL DISSECTION ALONG AVASCULAR EMBRYOLOGICAL PLANES}

The right part of the lesser sac is entered through the ontogenetic plane of the supramesocolic prepancreaticoduodenal omental fascia by detaching the omentum majus (the posterior aspect of the fourth leaflet of the greater omentum) from the underlining upper two-thirds of the anterior aspect of the pancreatic head, the anterior aspect of the second portion of the duodenum, and the anterior surface of the transverse mesocolon (colo-omental detachment). This is followed by lateromedial and craniomedial approaches to the submesocolic prepancreaticoduodenal area of Fredet. In detail, after freeing the hepatic flexure by dividing the hepatocolic and frenocolic ligaments, the cecum and right hemicolon are mobilized after incising the entire length of the colofascial interface. Following this, the dissection proceeds to the mesofascial interface, which enables a straightforward lateromedial-craniomedial detachment of the colon and small bowel mesentery from the retroperitoneal structures, along the fusion fascia of Toldt up to the ligament of Treitz, which, in continuity, is thoroughly prepared and completely divided. The right part of the root of the transverse mesocolon is carefully detached and mobilized medially down to the subisthmic mesenteric-portal vein axis by dissecting Fredet's area between the anterior leaf of the ascending and transverse mesocolon and the anterior aspect of the prepancreaticoduodenal fascia. Extending the dissection cephalad, the supra- and submesocolic prepancreaticoduodenal areas of dissection are unified, completely exposing the anterior surface of the pancreaticoduodenal complex. Dissection of Fredet's area is carried out with caution to safely dissect and divide Henle's venous trunk. An extended Lane-Kocher maneuver is then performed along the retropancreatic fusion fascia of Treitz up to the left side of the aorta, following division of the preduodenal laminae of Fruchaud.

Next, the intestinal rotation is reversed by rotating the in- testinal loop clockwise around the SMA axis, shifting the duodenojejunal junction and small intestine to the right of the SMA and the right hemi-colon to the left of the SMA (Fig. 2). Consequently, the SMA stem is displaced from left posteriorly to right posteriorly to the SMV (Fig. 3). Additionally, and most importantly, following this maneuver, the mesopancreas is unfolded to the right of the SMV-SMA axis and lies in a two-dimensional transverse plane.

The torsional arrangement between the pancreaticoduodenal complex and uppermost jejunum around the SMA axis is restored, shifting the IPDA and the JA1 (or the FJT) origins to the right aspect of the SMA. Accordingly, these arteries follow a right-lateral horizontal course (Fig. 3). Concurrently, the inferior pancreatic veins drain into the right aspect of the SMV or the FJPV coursing from right to left in a two-dimensional horizontal plane. With the completion of the Cattell-Braasch-Valdoni maneuver, the duodenojejunal junction forms an epsilon (ع) shape, lying in a two-dimensional horizontal plane and at the same time, the anatomy of the mesopancreas is simplified (Fig. 2). These features enable not only a safe and uncomplicated assessment of pancreatic head cancer resectability but also a straightforward and maximized mesopancreas excision.

The resection phase begins with the division of the proximal mesojejunum between the JA1 and the JA2 toward the SMA. The simplified arrangement of the proximal mesojejunum allows secure identification of the JA1 and the JA2. The prox-

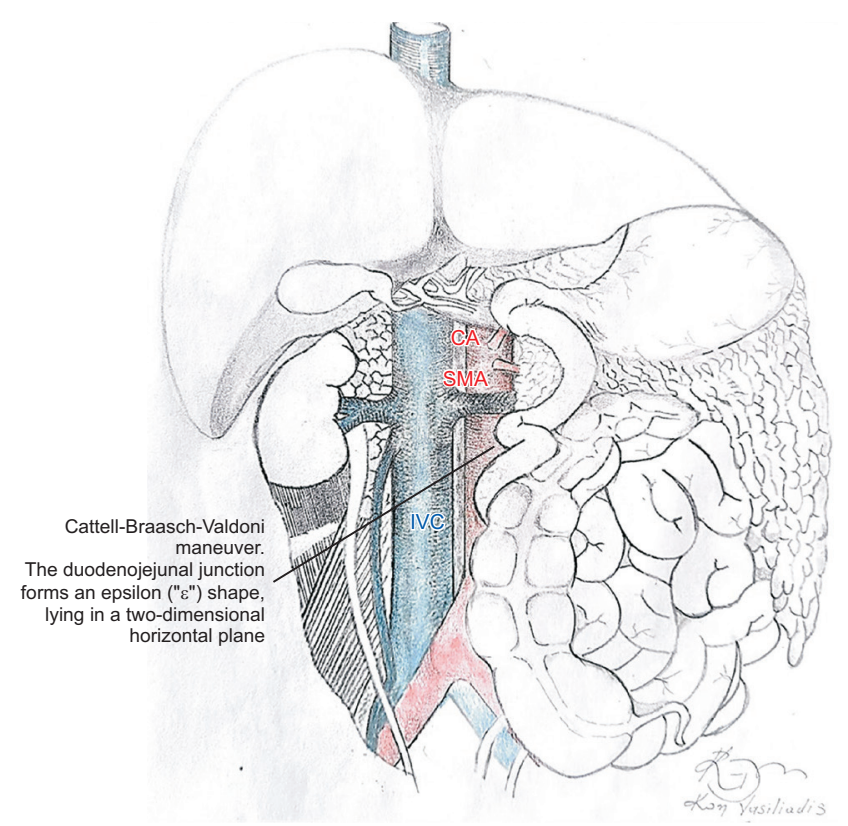

Fig. 2. The Cattell-Braasch-Valdoni maneuver. Derotation in a 270degree clockwise direction of the primary umbilical loop. It restores the embryologically twisted duodenojejunal junction by transposing the duodenum and uppermost jejunum to the right of the SMV-SMA axis in a two-dimensional horizontal plane. CA, celiac artery; SMA, superior mesenteric artery; IVC, inferior vena cava SMV, superior mesenteric vein. 


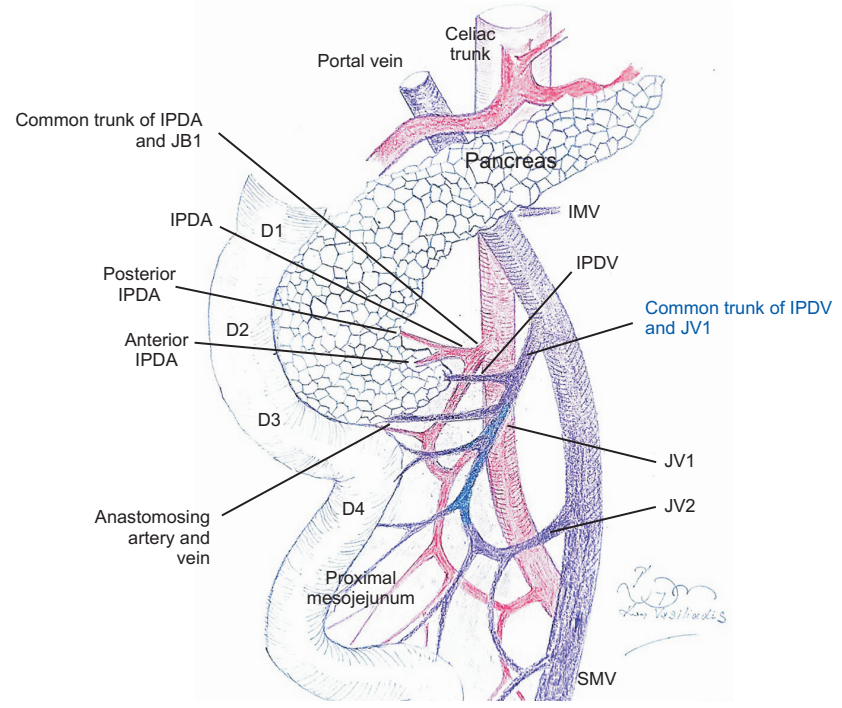

Fig. 3. Restoration of the torsional arrangement between the pancreaticoduodenal complex and uppermost jejunum around the SMA axis following the Cattell-Braasch-Valdoni maneuver. The IPDA and first jejunal artery origins are shifted to the right aspect of the SMA. The inferior pancreatic veins flow into the right aspect of the SMV or the first jejunopancreatic vein (FJPV), coursing from right to left in a twodimensional horizontal plane. IMV, inferior mesenteric vein; IPDV, inferior pancreaticoduodenal vein; IPDA, inferior pancreaticoduodenal artery; $J V$, jejunal vein; SMV, superior mesenteric vein; SMA, superior mesenteric artery.

imal mesentery is incised both at its anterior and posterior surfaces and the arcuate artery at the mesenteric border of the uppermost jejunum is identified and ligated. Next, the adipose mesenteric tissue is cautiously incised layer-by-layer toward the SMA. As the mesenteric incision proceeds toward the SMA, the FJPV is encountered, coursing parallel to the J2A. Several vein tributaries (anastomosing veins) from the pancreatic head and proximal mesojejunum flowing into the right aspect of the FJPV are carefully divided, while the FJPV is safeguarded and carefully preserved. At this level, the IPDV (usually defined as the main drainage vein of the uncinate process) is identified and encircled as it courses anteriorly to the SMA. As the mesenteric incision advances, the right aspect of the SMA is inevitably reached.

Following the identification of the right aspect of the SMA, plPh-II is carefully dissected and divided in a down-to-top approach (inferior-superior direction) along the right aspect of the SMA. As the dissection of plPh-II proceeds toward the origin of the SMA, the resectability of the tumor can be ultimately determined by clarifying the extent of tumor growth toward the anatomical components of plPh-II. Ventral traction of the pancreaticoduodenal complex can further facilitate the dissection of plPh-II.

At this phase of the operation, no irreversible step has been taken and in the case of unresectability, the procedure can be safely abandoned. Dissecting the fibrous layer investing the plPh-II, the IPDA and the JA1 (or the FJAT) are identified, arising from the right aspect of the SMA. In the case of resectability, the IPDA and the JA1 (or the FJAT) are divided, followed by the division of the main drainage vein of the uncinate process (usually defined as the IPDV).

It is very important to be aware that in many instances, the IPDV interferes with the origins of the IPDA and the JA1 or FJAT, making their identification and safe division almost impossible. In such a surgical scenario, the IPDV should be divided inevitably ahead of the division of the IPDA and JA1, allowing the straightforward identification and safe ligation of the arterial components of plPh-II.

As resectability has been determined, the gastroduodenal artery is divided and the duodenum, choledochus, pancreatic neck, and upper jejunum are transected. Following this, resection of the mesopancreas is performed. The venous tributaries flowing into the SMV and portal vein from the pancreatic head, including the vein of Belcher, are meticulously dissected and divided between the ligatures. The mobilization of the mesopancreas is continued cephalad along the right aspect of the SMA including plPh-I and the right part of the celiac ganglion at the level of the origin of the SMA and celiac artery (CA), clearing the anatomic area of the mesopancreas triangle.

In the case of definite resectability determined following the intestinal derotation maneuver, mesopancreas excision can be alternatively performed as the final step of the resection phase. It is very important to note that, at the end of the resection phase, the position of the intestines should be restored to their pre-derotation state without, however, the need for fixation sutures.

\section{DISCUSSION}

The right medial visceral rotation maneuver was originally reported by Cattell and Braasch [24] as a method to expose the third and fourth portions of the duodenum and was later modified by Valdoni in 1974 (Cattell-Braasch-Valdoni maneuver) [37]. It consists of the extensive lateromedial-craniomedial detachment and complete mobilization of the right hemi-colon and small bowel mesentery from the retroperitoneum along the fusion fascia of Toldt. This maneuver has eventually proven to be extremely useful in the exposure of the porto-mesenteric venous axis, pancreatic head, and retroperitoneal structures [11]. Apart from extensive colectomies and duodenal exposure, this maneuver is also currently indicated for vascular emergencies and retroperitoneal injuries [38]. However, since its original description in 1960, the Cattell-Braasch-Valdoni maneuver has limited dissemination in surgical practice.

During the last decade, there has been a number of important reports on the use and advantages of the Cattell-BraaschValdoni maneuver in pancreatic head cancer surgery [3941]. According to these reports, the underlying reason for the implementation of this maneuver in PD lies in the fact that it 
reverses the embryologically twisted duodenojejunal junction, creating a new favorable anatomical condition, enabling improved surgical exposure of the pancreaticoduodenal complex and proximal jejunum. This, in turn, entails a safer approach, more accurate clarification, and a deeper oncological clearance of the retropancreatic neurovascular bundle compared to conventional PD [11].

In 2016, Sugiyama et al. [25] first applied the intestinal derotation maneuver in artery-first approach $\mathrm{PD}$, targeting a safer and maximal mesopancreas excision. This maneuver facilitated extensive mesopancreas resection and allowed the early and safe control of blood flow into the pancreaticoduodenal complex. Similarly, in 2020, Akita et al. [26] reported that artery-first approach PD using the Cattell-Braasch maneuver simplified the anatomy around the SMA and SMV and enabled complete resection of the mesopancreas with more harvested lymph nodes in the SMA lymph node basin (station 14) compared to conventional PD.

The Cattell-Braasch-Valdoni maneuver succeeds in both accomplishing the wide exposure of the mesopancreas and straightforward clarification of the anatomy of the inferior peripancreatic vessels. These advantages become extremely important not only in the artery-first approach but also in conventional PD during the crucial surgical step of dissecting and clarifying the complicated anatomy of the vascular components of the mesopancreas.

A major concern when dissecting the inferior peripancreatic area is the identification and careful preservation of the FJPV. The FJPV usually drains a broad territory of the proximal mesojejunum corresponding to several proximal JAs and must, therefore, be carefully dissected and preserved to avoid massive bleeding and ensure the unrestrained venous return of the remaining jejunum $[42,43]$. However, in the rotation state (the normal anatomic state) the FJPV follows a twisted course predominately dorsally to the SMV and SMA [6,27-29]. This anatomic distortion makes the intraoperative clarification of the course, branching, and termination site of the FJPV extremely difficult and fraught with pitfalls. This is further complicated by the fact that the IPDV and a number of pancreatic and JV branches join the FJPV near its termination site on the SMV (or rarely on the splenoportal confluence). This anatomic complexity is resolved following the Cattell-Braasch-Valdoni maneuver. Indeed, as reported by both Sugiyama et al. [44] and Akita et al. [26], after intestinal derotation, the FJPV is positioned anterior-ventral to the SMA in the prevailing majority of cases, lying in a two-dimensional horizontal plane. This facilitates the secure preservation of the FJPV in addition to the clear identification and safe ligation of the IPDV and the venous branches coming from the uncinated process and proximal jejunum at their termination sites on the FJPV. Specifically, as shown in the study by Sugiyama et al. [44], following the Cattell-Braasch-Valdoni maneuver, the FJPV runs parallel to the JA2 between the layers of the proximal mesojejunum. This creates a favorable anatomic condition that facilitates the identification of the IPDV, which drains into the right wall of the FJPV or directly into the right aspect of the SMV. Additionally, the favorable anatomy created by intestinal derotation facilitates the identification of the pancreatic and JV branches that join the right aspect of the FJPV.

The Cattell-Braasch-Valdoni maneuver also facilitates the secure identification of the arterial components of the mesopancreas. In fact, as shown in the study by Akita et al. [26], following intestinal derotation, the roots of the IPDA, the JA1 (or the FJT), and proximal JAs, which in the normal anatomic state, branch off from the dorsal or left aspect of the SMA, are shifted to the right aspect of the SMA and run a right-lateral horizontal course (Fig. 3). Therefore, they can be easily dissected in a flat plane.

Essentially, the Cattell-Braasch-Valdoni maneuver shifts the dorsal aspect of the SMA to the right, which adequately exposes the orifices and demystifies the branching pattern of the IPDA, the JA1 (or the FJT), and the proximal JAs, facilitating dissection toward the root of the SMA. It should be emphasized that this advantage enables the secure identification and safe ligation of the arterial components of the mesopancreas, regardless of their branching pattern. Actually, considering the complex, understudied, and variant inferior peripancreatic vascular anatomy, it is not hyperbolic to argue that without the Cattell-Braasch-Valdoni maneuver it would be extremely unlikely to correctly clarify the anatomy of the mesopancreas and identify the vein components of the FJPV and the arterial tributaries of the FJT, which, in the normal anatomical state, are arranged in a twisted configuration behind the superior mesenteric vessels.

However, it should be noted that even after intestinal derotation, the venous components of a ventrally-to-SMA located FJPV may hide the origins of the IPDA-JA1 or the FJAT (despite the fact that their orifices are shifted to the right aspect of the SMA), making their identification and safe ligation practically impossible. Indeed, as reported by Sugiyama et al. [44], after intestinal derotation, the FJPV is situated anterior to the SMA in $91 \%$ of the cases, and the IPDV may interfere with the origins of the IPDA and JA1 or FJAT. In such an operative scenario, the IPDV should be divided ahead of the division of the IPDA and JA1, allowing the straightforward identification and safe ligation of the arterial components of the mesopancreas. Considering the dangerous variations of the branching pattern of the proximal jejunal vessels such as Ishikawa type 3 [28], and/or Kobayashi types Ib, Ic IIb, IIc, and IId [33], the CattellBraasch-Valdoni maneuver offers the best chance to correctly clarify the anatomy of the mesopancreas and overcome the surgical pitfalls related to the dissection of the inferior peripancreatic area.

In essence, the Cattell-Braasch-Valdoni maneuver dissociates the embryological concrescence planes fixing the pancreaticoduodenal complex and proximal jejunum to the 
derivatives of the dorsal mesogastrium and dorsal parietal wall (deep subperitoneal fascia) and completely derotates the primary midgut loop, eliminating the complicated developmental distortion of the mesopancreas, which, following derotation, unfolds mediolaterally and lies in a two-dimensional horizontal plane. Ultimately, this maneuver provides "mesenterization" of the mesopancreas, which converts from a complex three-dimensional into a simpler two-dimensional structure similar to that of the small bowel mesentery. This constitutes the main advantage of the Cattell-Braasch-Valdoni maneuver, namely, demystification of the vascular anatomy of the mesopancreas, regardless of branching pattern variations. Equally important is that this maneuver enables a straightforward and safe maximal mesopancreas excision.

Additionally, as shown in the study of Sugiyama et al. [25], the Cattell-Braasch-Valdoni maneuver facilitates the early assessment of resectability and enables the early exposure and ligation of the IPDA. Furthermore, it decreases operative time and blood loss and improves the rate of radical resections of pancreatic head cancer compared to conventional PD without increasing the postoperative morbidity rate [25].

The Cattell-Braasch-Valdoni maneuver is also useful in the case of a replaced right hepatic artery (RHA) originating from the SMA. This rather frequent anatomical variation [45] increases the difficulty of mesopancreas excision because it interferes with the SMA resection margin [46]. The CattellBraasch-Valdoni maneuver allows for the early identification and preservation of a replaced RHA originating from the SMA, favoring a safe and maximal mesopancreas excision.

Special attention is warranted to the usefulness of the Cattell-Braasch-Valdoni maneuver in the case of radical excision of borderline resectable pancreatic cancer that necessitates venous resection and reconstruction. Especially in cases that require more than $5 \mathrm{~cm}$ of venous segment resection, the available reconstruction options (autologous, heterologous, or polytetrafluethylene graft) have significant specific drawbacks, making their use problematic $[40,47,48]$. Contrary to these methods, the Cattell-Braasch-Valdoni maneuver can be easily performed, allowing a safe and tension-free direct end-to-end venous anastomosis, regardless of the length of the resected venous segment [49] since the distal SMV stump can easily be shifted cranially because the root of the small bowel mesentery is completely mobilized [50].

In conclusion, the Cattell-Braasch-Valdoni maneuver can be safely and easily associated with $\mathrm{PD}$, simplifying the peripancreatic anatomical field and allowing the straightforward and maximized resection of the mesopancreas, which results in a higher $\mathrm{R} 0$ resection rate. Therefore, it should be considered as a valuable approach in mesopancreas-first pancreatic head cancer resection for both resectable and borderline resectable tumors. Further investigation is warranted to validate the usefulness and effectiveness of this master technique in pancreatic head cancer resection.

\section{CONFLICT OF INTEREST}

No potential conflict of interest relevant to this article was reported.

\section{REFERENCES}

1. Kimura W. Surgical anatomy of the pancreas for limited resection. J Hepatobiliary Pancreat Surg 2000;7:473-479.

2. Gaedcke J, Gunawan B, Grade M, Szöke R, Liersch T, Becker H, et al. The mesopancreas is the primary site for $\mathrm{R} 1$ resection in pancreatic head cancer: relevance for clinical trials. Langenbecks Arch Surg 2010;395:451-458.

3. Esposito I, Kleeff J, Bergmann F, Reiser C, Herpel E, Friess H, et al. Most pancreatic cancer resections are R1 resections. Ann Surg Oncol 2008;15:1651-1660.

4. Adham M, Singhirunnusorn J. Surgical technique and results of total mesopancreas excision (TMpE) in pancreatic tumors. Eur J Surg Oncol 2012;38:340-345.

5. Peparini N, Chirletti P. Clearance of the retropancreatic margin in pancreatic carcinomas: total mesopancreas excision or extended lymphadenectomy? Eur J Surg Oncol 2012;38:1146; author reply 1147.

6. Inoue Y, Saiura A, Yoshioka R, Ono Y, Takahashi M, Arita J, et al. Pancreatoduodenectomy with systematic mesopancreas dissection using a supracolic anterior artery-first approach. Ann Surg 2015;262:1092-1101.

7. Neoptolemos JP, Palmer DH, Ghaneh P, Psarelli EE, Valle JW, Halloran $\mathrm{CM}$, et al. Comparison of adjuvant gemcitabine and capecitabine with gemcitabine monotherapy in patients with resected pancreatic cancer (ESPAC-4): a multicentre, open-label, randomised, phase 3 trial. Lancet 2017;389:1011-1024.

8. Sharma D, Isaji S. Mesopancreas is a misnomer: time to correct the nomenclature. J Hepatobiliary Pancreat Sci 2016;23:745-749.

9. Peparini N. Mesopancreas: a boundless structure, namely the rationale for dissection of the paraaortic area in pancreaticoduodenectomy for pancreatic head carcinoma. World J Gastroenterol 2015;21:2865-2870.

10. Hartwig W, Vollmer CM, Fingerhut A, Yeo CJ, Neoptolemos JP, Adham M, et al.; International Study Group on Pancreatic Surgery. Extended pancreatectomy in pancreatic ductal adenocarcinoma: definition and consensus of the International Study Group for Pancreatic Surgery (ISGPS). Surgery 2014;156:1-14.

11. Borghi F, Gattolin A, Garbossa D, Bogliatto F, Garavoglia M, Levi AC. Embryologic bases of extended radical resection in pancreatic cancer. Arch Surg 1998;133:297-301.

12. Hagai H. Configurational anatomy of the pancreas: its surgical relevance from ontogenetic and comparative-anatomical viewpoints. J Hepatobiliary Pancreat Surg 2003;10:48-56.

13. Isaji S, Murata Y, Kishiwada M. New Japanese classification of pancreatic cancer. In: Neoptolemos J, Urrutia R, Abbruzzese J, Büchler M, eds. Pancreatic cancer. New York: Springer, 2018:1021-1037.

14. Schneider M, Strobel O, Hackert T, Büchler MW. Pancreatic resection for cancer-the Heidelberg technique. Langenbecks Arch Surg 
2019;404:1017-1022.

15. Japan Pancreas Society. Classification of pancreatic carcinoma. 2nd English edition. Tokyo: Kanehara, 2003.

16. Peparini N. Para-aortic dissection in pancreaticoduodenectomy with mesopancreas excision for pancreatic head carcinoma: not only an N-staging matter. J Gastrointest Surg 2016;20:1080-1081.

17. Ohigashi H, Ishikawa O, Eguchi H, Yamada T, Sasaki Y, Noura S, et al. Early ligation of the inferior pancreaticoduodenal artery to reduce blood loss during pancreaticoduodenectomy. Hepatogastroenterology $2004 ; 51: 4-5$.

18. Weitz J, Rahbari N, Koch M, Büchler MW. The "artery first" approach for resection of pancreatic head cancer. J Am Coll Surg 2010;210:e1-e4.

19. Hackert T, Werner J, Weitz J, Schmidt J, Büchler MW. Uncinate process first--a novel approach for pancreatic head resection. Langenbecks Arch Surg 2010;395:1161-1164.

20. Pessaux P, Varma D, Arnaud JP. Pancreaticoduodenectomy: superior mesenteric artery first approach. J Gastrointest Surg 2006;10:607-611.

21. Varty PP, Yamamoto H, Farges O, Belghiti J, Sauvanet A. Early retropancreatic dissection during pancreaticoduodenectomy. Am J Surg 2005;189:488-491.

22. Horiguchi A, Ishihara S, Ito M, Nagata H, Shimizu T, Furusawa K, et al. Pancreatoduodenectomy in which dissection of the efferent arteries of the head of the pancreas is performed first. J Hepatobiliary Pancreat Surg 2007;14:575-578.

23. Xu J, Tian X, Chen Y, Ma Y, Liu C, Tian L, et al. Total mesopancreas excision for the treatment of pancreatic head cancer. J Cancer 2017;8:3575-3584.

24. Cattell RB, Braasch JW. A technique for the exposure of the third and fourth portions of the duodenum. Surg Gynecol Obstet 1960;111:378379.

25. Sugiyama M, Suzuki Y, Nakazato T, Yokoyama M, Kogure M, Abe N, et al. Intestinal derotation procedure for facilitating pancreatoduodenectomy. Surgery 2016;159:1325-1332.

26. Akita M, Yamasaki N, Miyake T, Mimura K, Maeda E, Nishimura T, et al. Cattell-Braasch maneuver facilitates the artery-first approach and complete excision of the mesopancreas for pancreatoduodenectomy. J Surg Oncol 2020;121:1126-1131.

27. Papavasiliou P, Arrangoiz R, Zhu F, Chun YS, Edwards K, Hoffman JP. The anatomic course of the first jejunal branch of the superior mesenteric vein in relation to the superior mesenteric artery. Int J Surg Oncol 2012;2012:538769.

28. Ishikawa Y, Ban D, Matsumura S, Mitsunori Y, Ochiai T, Kudo A, et al. Surgical pitfalls of jejunal vein anatomy in pancreaticoduodenectomy. J Hepatobiliary Pancreat Sci 2017;24:394-400.

29. Sakaguchi T, Suzuki S, Morita Y, Oishi K, Suzuki A, Fukumoto K, et al. Analysis of anatomic variants of mesenteric veins by 3 -dimensional portography using multidetector-row computed tomography. Am J Surg 2010;200:15-22.

30. Horiguchi A, Ishihara S, Ito M, Nagata H, Asano Y, Yamamoto T, et al. Multislice CT study of pancreatic head arterial dominance. J Hepatobiliary Pancreat Surg 2008;15:322-326.

31. Horiguchi A, Ishihara S, Ito M, Asano Y, Yamamoto T, Miyakawa
S. Three-dimensional models of arteries constructed using multidetector-row CT images to perform pancreatoduodenectomy safely following dissection of the inferior pancreaticoduodenal artery. J Hepatobiliary Pancreat Sci 2010;17:523-526.

32. Negoi I, Beuran M, Hostiuc S, Negoi RI, Inoue Y. Surgical anatomy of the superior mesenteric vessels related to pancreaticoduodenectomy: a systematic review and meta-analysis. J Gastrointest Surg 2018;22:802-817.

33. Kobayashi Y, Sakamoto Y, Arita J, Akamatsu N, Kaneko J, Hasegawa $\mathrm{K}$, et al. Vascular anatomy of the jejunal mesentery and complications associated with division of the first jejunal venous trunk during pancreaticoduodenectomy. J Surg Oncol 2018;117:1297-1304.

34. Hongo N, Mori H, Matsumoto S, Okino Y, Ueda S, Shuto R. Anatomical variations of peripancreatic veins and their intrapancreatic tributaries: multidetector-row CT scanning. Abdom Imaging 2010;35:143153.

35. Yamada Y, Mori H, Kiyosue H, Matsumoto S, Hori Y, Maeda T. CT assessment of the inferior peripancreatic veins: clinical significance. AJR Am J Roentgenol 2000;174:677-684.

36. Kim HJ, Ko YT, Lim JW, Lee DH. Radiologic anatomy of the superior mesenteric vein and branching patterns of the first jejunal trunk: evaluation using multi-detector row CT venography. Surg Radiol Anat 2007;29:67-75.

37. Valdoni P. Chirurgia addominale: tecniche operatorie. Milano: Casa Editrice Francesco Vallardi, 1974.

38. Tocchi A, Mazzoni G, Puma F, Miccini M, Cassini D, Bettelli E, et al. Adenocarcinoma of the third and fourth portions of the duodenum: results of surgical treatment. Arch Surg 2003;138:80-85.

39. Bechi P, Maltinti G. A novel "salvage" indication to intestinal derotation procedure: reconstruction after duodenopancreatectomy. Am J Case Rep 2014;15:322-325.

40. Del Chiaro M, Segersvärd R, Rangelova E, Coppola A, Scandavini $\mathrm{CM}$, Ansorge C, et al. Cattell-Braasch maneuver combined with artery-first approach for superior mesenteric-portal vein resection during pancreatectomy. J Gastrointest Surg 2015;19:2264-2268.

41. Virgilio E, Amodio PM, Scorsi A, Goglia A, Macarone Palmieri R. Advantages of the maneuver of intestinal derotation for pancreaticoduodenectomy. J Invest Surg 2016;29:359-365.

42. Nakamura M, Nakashima H, Tsutsumi K, Matsumoto H, Muta Y, Ueno D, et al. First jejunal vein oriented mesenteric excision for pancreatoduodenectomy. J Gastroenterol 2013;48:989-995.

43. Katz MH, Fleming JB, Pisters PW, Lee JE, Evans DB. Anatomy of the superior mesenteric vein with special reference to the surgical management of first-order branch involvement at pancreaticoduodenectomy. Ann Surg 2008;248:1098-1102.

44. Sugiyama M, Suzuki Y, Nakazato T, Yokoyama M, Kogure M, Matsuki R, et al. Vascular anatomy of mesopancreas in pancreatoduodenectomy using an intestinal derotation procedure. World J Surg 2020;44:3441-3448.

45. Dandekar U, Dandekar K, Chavan S. Right hepatic artery: a cadaver investigation and its clinical significance. Anat Res Int 2015;2015:412595.

46. Jah A, Jamieson N, Huguet E, Praseedom R. The implications of the 
presence of an aberrant right hepatic artery in patients undergoing a pancreaticoduodenectomy. Surg Today. 2009;39:669-674.

47. Takasaki K, Yamamoto M, Tsugita M, Ootsubo T, Imaizumi T, Yoshikawa T. N Nine-year patency of a vascular prosthesis used for portal vein reconstruction during pancreatoduodenectomy. J Hepatobiliary Pancreat Surg 1997;4:127-130.

48. Herscu G, Wilson SE. Prosthetic infection: lessons from treatment of the infected vascular graft. Surg Clin North Am 2009;89:391-401, viii.

49. Fujisaki S, Tomita R, Fukuzawa M. Utility of mobilization of the right colon and the root of the mesentery for avoiding vein grafting during reconstruction of the portal vein. J Am Coll Surg 2001;193:576-578.

50. Muttillo EM, Felli E, Pessaux P. Cattell-Braasch maneuver in pancreatic surgery. No need of venous graft for vascular resection. J Surg Oncol 2020;122:1612-1615. 